\title{
Corela
}

Cognition, représentation, langage

HS-35 | 2022

Anaphore et pronoms en anglais : convergences, différences et complémentarité de quelques approches linguistiques

\section{Les pronoms : quelques problèmes de délimitation de la catégorie}

Jérôme Puckica

\section{OpenEdition \\ Journals}

Édition électronique

URL : https://journals.openedition.org/corela/14362

DOI : 10.4000/corela. 14362

ISSN : 1638-573X

Éditeur

Cercle linguistique du Centre et de l'Ouest - CerLICO

Référence électronique

Jérôme Puckica, «Les pronoms : quelques problèmes de délimitation de la catégorie », Corela [En ligne], HS-35 | 2022, mis en ligne le 04 janvier 2022, consulté le 24 janvier 2022. URL : http:// journals.openedition.org/corela/14362 ; DOI : https://doi.org/10.4000/corela.14362

Ce document a été généré automatiquement le 24 janvier 2022.

\section{(c) (1)(2)}

Corela - cognition, représentation, langage est mis à disposition selon les termes de la licence Creative Commons Attribution - Pas d'Utilisation Commerciale - Partage dans les Mêmes Conditions 4.0 International. 


\title{
Les pronoms : quelques problèmes de délimitation de la catégorie
}

\author{
Jérôme Puckica
}

1 La classe des pronoms est une des plus anciennes classes de mots de la tradition grammaticale occidentale. Elle figurait déjà parmi les huit « parties de la phrase » de la

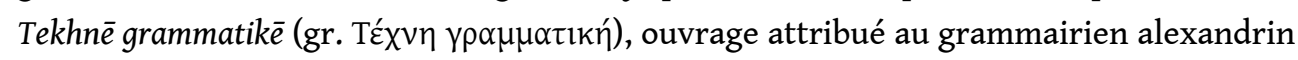
Denys le Thrace (170-90 av. J-.C) et souvent considéré comme la plus ancienne grammaire en Occident (cf. Lallot 1998). Apollonios Dyscole, le grand grammairien alexandrin du début du 2e s., a quant à lui consacré un traité entier aux pronoms (Пвpì

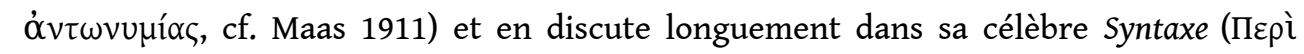

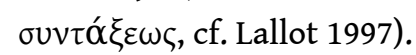

2 En dépit de son ancienneté, la classe des pronoms demeure une des classes de mots les plus problématiques et ce, dans de nombreuses langues. En anglais, se trouvent ainsi fréquemment regroupés sous l'appellation pronom des mots qui n'ont en définitive que très peu de choses en commun, tels $I$, what?, everything, many, one(s) et (the) same. A toutes fins utiles, nous donnons ci-dessous une liste conventionnelle des principales sous-classes de pronoms traditionnellement reconnues en anglais (1) avec quelques exemples, incluant certains mots dont nous arguerons plus tard qu'ils ne devraient en fait pas être classés parmi les pronoms.
(1) Principales sous-classes de pronoms traditionnellement reconnues en anglais
a. PERSONAL: I/me, you, he/him, she/her, it, we/us, they/them

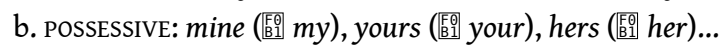
c. REFLEXIVE: myself, yourself, herself...
d. RECIPROCAL: each other, one another
e. DEMONSTRATIVE: this/these, that/those
f. INTERROGATIVE: who( $m)$, what, which; whoever, whichever, whatever
g. RELATIVE: who(m), which, what; whoever, whatever; that
h. INDEFINITE: something, anything, no one, everybody...; all, both; some, any, much, many,
few, several, most, each, (n)either, one ${ }_{\text {gen }}$; one (s), other(s); (the) same, (the) last...

3 Face à cet ensemble hétérogène de formes, il convient de s'interroger tout d'abord quant à la définition que l'on peut donner de la classe des pronoms. La définition 
traditionnelle du pronom est inscrite dans l'étymologie du mot : le pro-nom s'emploie " pour un nom ", «à la place d'un nom ». Nous commencerons par un examen de cette définition étymologique qui, comme on le sait, est loin d'être satisfaisante (\$1). Dans un deuxième temps, nous proposerons de redéfinir les pronoms comme une sous-classe fermée de noms grammaticaux qui ne prennent pas de déterminant et dont les membres centraux s'emploient typiquement de façon anaphorique ou déictique, les membres les plus centraux de la classe étant bien entendu les pronoms personnels (\$2). Enfin, nous nous intéresserons à la distinction catégorielle entre les pronoms et les déterminants, distinction controversée et même souvent réfutée (\$3). Nous suggèrerons qu'il est préférable de maintenir cette distinction au moins pour les articles et les pronoms personnels, tout en reconnaissant que de nombreux « couples » déterminant-pronom (ex. Read this message vs Read this) prêtent à discussion.

\section{La définition étymologique du « pro-nom »}

Suivant une formule qui remonte à l'Antiquité grecque et a été reprise (et critiquée) par des générations de grammairiens jusqu'à nos jours, le pronom est un mot qui est mis " pour un nom ", s'emploie " à la place d'un nom », « remplace un nom », etc., d'où son appellation de pronom (angl. pronoun, alld. Pronomen ou Fürwort, esp. pronombre, it. pronome, etc.), du lat. prōnōmen, calque du grec ancien $\alpha \dot{\alpha} \tau \omega v v \mu i^{\alpha} .{ }^{1}$

5 L'étymologie du mot pronom est en fait très ambiguë (cf. n. 2), mais suivant l'interprétation courante, c'est un mot dont la fonction fondamentale serait la « reprise » anaphorique d'un nom. Comme le note Lyons $(1977: 657):$ « the term 'pronoun' owes its origin to the view that there are certain forms or expressions whose function it is to operate as substitutes for nouns ». De même, écrivent Gardes Tamine et al. (2015 : 248) : "Du latin pro nomen, " mis pour le nom» (mais en réalité mis pour le syntagme nominal, autrement dit le nom actualisé en discours), le pronom a fondamentalement vocation à permettre la reprise d'un élément déjà cité, lequel constitue alors son antécédent. »

Cette définition étymologique du pronom pose évidemment plusieurs problèmes, dont les trois suivants : (i) seuls certains pronoms peuvent être anaphoriques; (ii) l'antécédent d'un pronom n'est pas toujours un nom ; (iii) l'anaphore n'est pas un " remplacement ».

\subsection{Pronoms et anaphore}

7 Seuls certains pronoms sont ou peuvent être anaphoriques, un pronom étant dit d'emploi anaphorique lorsque son référent est identifié grâce à un antécédent dans le contexte linguistique :

(2) a. I asked Jenny if she knew the answer.

b. When I first met him, Sven worked for the German embassy.

c. That's your problem, not mine.

d. The man who gave his paycheque to his wife was wiser than the man who gave it

to his mistress. (Karttunen 1969)

8 Dans le cas le plus élémentaire, l'antécédent précède le pronom et les deux formes sont coréférentielles, comme dans l'interprétation la plus simple de (2a) - la plus simple, puisque she pourrait éventuellement désigner une autre personne que Jenny. Sans 
entrer ici dans une discussion de l'anaphore, notons que plusieurs autres cas sont possibles. Par exemple, il arrive que l'antécédent, malgré son nom, suive le pronom, comme dans l'interprétation la plus simple de (2b). Pour cette raison, on distingue souvent l'anaphore, dans un sens alors plus restreint du terme (« antécédent placé avant»), de la cataphore ("antécédent placé après »). Par défaut, cependant, anaphorique signifiera ici ana- ou cataphorique et sera ainsi synonyme de endophorique au sens de Halliday \& Hasan (1976). Parmi d'autres cas possibles, il arrive que l'antécédent et le pronom ne soient pas coréférentiels (2c-d), que le pronom ne soit que partiellement anaphorique (ex. (4) infra), que l'antécédent ne soit pas clairement identifiable (ex. (8)), que la référentialité du pronom (et avec elle, son éventuelle anaphoricité) soit incertaine, etc.

9 L'emploi anaphorique est typique des pronoms personnels, possessifs et réfléchis de $3 \mathrm{e}$ personne. Il est également courant avec les réciproques ou encore les démonstratifs, mais ces derniers peuvent aussi avoir un fonctionnement déictique (3a-b) - le latin

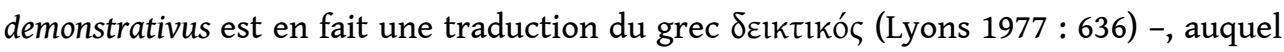
cas ils désignent directement un élément du contexte situationnel, sans antécédent dans le contexte linguistique. Ce fonctionnement déictique est tout particulièrement caractéristique des pronoms personnels de 1e et $2 \mathrm{e}$ p. (3c), qui désignent le locuteur (énonciateur) ou l'allocutaire (co-énonciateur), ou, au pluriel, un groupe qui les inclut :

(3) a. Look at that! [pointant vers quelque chose de visible dans la situation]

b. Isn't this the key you were looking for? [montrant la clef à l'allocutaire]

c. I'm telling you that she lied.

d. 'What happened to him?', she asked, pointing to one of the guards.

10 Le terme pronom étant censé évoquer une reprise anaphorique, il est notable que les pronoms de $1 \mathrm{e}$ et $2 \mathrm{e} \mathrm{p}$. - les plus fondamentaux d'entre tous, dans la mesure où tout acte d'énonciation requiert un locuteur et instaure plus ou moins explicitement un allocutaire - n'admettent que très difficilement un emploi anaphorique. ${ }^{2} \mathrm{~A}$ l'inverse, les pronoms personnels de $3 \mathrm{e} \mathrm{p}$. se prêtent assez mal à des emplois déictiques, sans pour autant les exclure (3d).

11 La distinction entre anaphore et deixis, cependant, est souvent loin d'être claire, car il s'agit là de deux modes de fonctionnement qui ne sont pas mutuellement exclusifs. Dans (3d), par exemple, him est a priori déictique pour les interlocuteurs du discours rapporté - les personnages de la scène -, mais anaphorique pour le lecteur, qui ne peut accéder à la scène qu'à travers le texte. Comme le note aussi Corblin (1995: 17) en français : " loin de s'exclure, les deux usages [i.e. anaphorique et déictique] sont dans beaucoup de situations indistinguables. [...] Ainsi, supposons qu'un locuteur présente quelqu'un à son interlocuteur en disant: [Voici Monsieur X. Il sera votre assistant.] Il n'est guère alors possible de trancher entre l'usage anaphorique et l'usage déictique de il, la présentation de l'individu coïncidant avec sa mention. " De plus, certaines formes peuvent être à la fois anaphoriques et déictiques, comme dans les exemples suivants de Stirling \& Huddleston $(2002: 1454)$ :

(4) a. Sue is coming over later; we are having lunch together.

b. I was born in London and have lived here all my life.

12 Dans (4a), we peut désigner le groupe constitué par le locuteur et Sue, auquel cas ce pronom est partiellement déictique - il renvoie en partie au locuteur - et partiellement anaphorique - l'identité de l'autre personne est déterminée via Sue, qui sert d'antécédent. Dans (4b), de même, here est à la fois déictique - here désigne l'endroit où 
se trouve le locuteur - et anaphorique - l'endroit est compris comme étant Londres, in London servant d'antécédent.

Si certains pronoms sont typiquement anaphoriques ou déictiques, de nombreux autres ne peuvent être ni l'un, ni l'autre, dont les indéfinis composés en some-, any-, no- + body, -one, -thing (5a-b), le pronom générique one (5c), les interrogatifs what? et who? (5d-e), ou encore le « relatif indéfini » whoever (5f) :

(5) a. Everybody knows the fight was fixed.

b. Someone called.

c. One can never be too careful.

d. What happened?

e. Who called?

f. Whoever did this knew where to find the key.

Enfin, et inversement, les formes anaphoriques ou déictiques ne sont pas toutes des pronoms. On peut considérer que les pronoms font partie d'une classe fonctionnelle plus large de proformes qui inclut des mots de diverses catégories, dont le verbe « de reprise » do (6a), certains adverbes (6b-d), adjectifs (6e), déterminants (6f), et même des noms dénombrables dans le cas de one(s) et other $(s)(6 \mathrm{~g})$, dont il sera discuté plus loin (cf. \$2.3). ${ }^{3}$

(6) a. I worry more about her than I do about you.

b. She went to London and stayed there ten days.

c. The bell struck 10 p.m. Only then did he realise that something was amiss.

d. She reacted very strangely, and so did he.

e. There is no such thing as a good war.

f. There was an umbrella on the table but that umbrella was not mine.

g. The other room is just like this one.

15 L'appellation proforme (angl. pro-form) est souvent limitée aux seules formes anaphoriques (ex. Quirk et al. 1985, Trask 1993, Leech 2006), mais il semblerait plus cohérent de l'élargir aux formes déictiques, d'autant que certains des mots concernés peuvent avoir les deux fonctionnements, voire les deux à la fois. En outre, puisque des mots tels que who?, whoever et anything sont classés parmi les pronoms alors qu'ils ne sont ni anaphoriques, ni déictiques, on pourrait juger que des adverbes tels que when?, how?, wherever et anywhere ont également leur place parmi les proformes.

\subsection{Nature de l'antécédent}

Contrairement à ce que suggère l'étymologie du terme, l'antécédent d'un pro-nom n'est pas toujours un nom. C'est le cas dans (2a-b), mais, bien souvent, il s'agit d'un GN, comme dans (2c-d). Pour cette raison, on dit souvent du pronom qu'il porte mal son nom : « pronoun is a misnomer [...] a pronoun tends to be a surrogate for a whole noun phrase rather than a noun » (Quirk et al. $1985: 76$ ) ; « pronouns replace noun phrases, not nouns - and should more accurately be called pro-NPs » (Börjars \& Burridge 2010 : 167).

L'antécédent d'un pronom n'est parfois même pas une expression nominale, mais une proposition ou une phrase :

(7) a. I knew she would be late again. I knew it!

b. I'm sorry to tell you this but your husband is an idiot.

c. A: 'I've never learned to swim.' - B: 'That's a pity.' 

large, voire difficile à cerner de façon précise - un antécédent « vague ». On peut ainsi se demander quel est exactement l'antécédent de it ou this dans le passage suivant :

(8) That was ' 68 , back when the wild behavior was still new. People suddenly forced to make sense of madness. All that public display. The dropping of inhibitions. Authority powerless. The kids going crazy. Intimidating everybody. The adults don't know what to make of it, they don't know what to do. Is this an act? Is the 'revolution' real? Is it a game? Is it cops and robbers? What's going on here? [...] (P. Roth, American Pastoral, 1997, US)

De façon plus discutable, (the) same est parfois considéré comme un pronom dont l'antécédent peut être adjectival ou même prépositionnel :

(9) a. The soup smells delicious, and the turkey smells the same.

$\mathrm{a}^{\prime}$. The soup smells delicious, and the turkey smells exactly the same.

b. Yesterday I felt under the weather, and today I feel the same.

Selon Quirk et al. (1985 : 873), à qui nous empruntons les exemples (9a) et (9b), la proforme the same aurait pour antécédent l'adjectif delicious dans (9a) et le GP under the weather dans (9b). ${ }^{4}$ Toutefois, le caractère anaphorique de cette expression est incertain et l'on peut se demander s'il ne s'agit pas simplement d'un emploi de l'adjectif same exprimant l'identité. Dans (9a'), par exemple, où exactly a été ajouté devant the same, le sens est bien «exactly identical », pas « exactly delicious » : c'est l'identité de l'odeur qui est parfaite, pas l'odeur elle-même. The same exprime une notion d'identité ou similarité, qui implique la comparaison d'une entité (repérée) par rapport à une autre (repère), mais cela n'en fait pas nécessairement un pronom ou une proforme anaphorique. Dans certains emplois, comme All people are the same et They were both treated the same, aucun antécédent ne peut d'ailleurs être reconnu et seule demeure la notion d'identité, avec un remplacement possible de the same par identical ou identically, selon l'énoncé.

\subsection{Anaphore vs remplacement}

Enfin, il est trompeur, voire inexact, de dire qu'un pronom anaphorique est « mis à la place » de son antécédent, le "remplace » ou s'y « substitue ». Une telle présentation est particulièrement inappropriée lorsque le pronom et l'antécédent ne sont pas coréférentiels :

(10) Jenny has got her own plan and I've got \{mine / *her own plan\}.

Parler de remplacement demeure contestable même lorsque le pronom et l'antécédent sont coréférentiels. En effet, dire que $B$ remplace $A$ dans la position $X$ suggère que $A$ pourrait occuper $\mathrm{X}$. Or, la relation entre un pronom anaphorique et son antécédent est typiquement à sens unique, dans la mesure où le "remplacé " (antécédent) ne peut généralement pas être mis à la place du " remplaçant » (pronom) $:^{5}$

(11) $a$. I asked Jenny $y_{\mathrm{i}}$ if she $\mathrm{i}_{\mathrm{i}}$ knew the answer. [= (2a)]

b. ${ }^{*}$ I asked Jenny ${ }_{i}$ if Jenny ${ }_{i}$ knew the answer.

De même, si le sujet et l'objet d'un verbe sont coréférentiels, alors l'objet doit être un pronom réfléchi, qui ne peut donc pas être remplacé par son antécédent :

(12) a. The barber shaved himself. $_{i}$.

b. *The barber shaved the barber $_{\mathrm{i}}$. 

formuler une définition tout à fait satisfaisante de l'ensemble des mots qui sont habituellement nommés pronoms (cf. (1)). Les pronoms personnels ont un statut prééminent au sein de la classe des pronoms, mais l'appellation pronom a été étendue à des mots qui diffèrent considérablement des pronoms personnels, de sorte qu'il n'y a en définitive que très peu de propriétés communes à l'ensemble des pronoms de la tradition : ils peuvent être morphologiquement simples (you) ou complexes (each other) ; variables (this/these) ou invariables (something) ; définis (she), indéfinis (someone), voire ni l'un ni l'autre ((the) others); typiquement anaphoriques (she), déictiques (you), 
ou à nouveau ni l'un ni l'autre (whoever)... Enfin, les pronoms sont des sortes de noms qui forment ensemble une classe fermée et qui, de façon caractéristique, ne prennent pas de déterminant ( ${ }^{*}$ a you ${ }^{*}$ the this), mais il y là encore des exceptions, telles que one et others - deux mots qui n'ont pas leur place parmi les pronoms, comme nous allons le voir.

Tenant compte de ces facteurs, et suivant notamment Hudson $(1990,2007)$ et Payne \& Huddleston (2002), nous proposerons ici la redéfinition (16), dont divers aspects sont expliqués ci-après :

(16) Les pronoms sont une sous-classe fermée de noms qui ne prennent pas de déterminant, dont les membres centraux fonctionnent typiquement de façon anaphorique ou déictique.

\subsection{Les pronoms personnels comme prototype catégoriel}

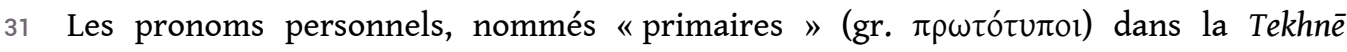
grammatikē, semblent toujours avoir été au centre des réflexions sur les pronoms et apparaissent de fait comme les pronoms les plus fondamentaux d'entre tous. En d'autres termes, les pronoms semblent former une catégorie prototypique dont les membres les plus centraux sont les pronoms personnels, et plus précisément ce qu'on peut appeler les pronoms personnels au sens étroit, ou " personnels ${ }_{1}$ ", i.e. en anglais, les formes au cas sujet et/ou complément que sont : I/me, you, he/him, she/her, it, we/us, they/them. Ces pronoms constituent eux-mêmes une sous-classe des pronoms personnels au sens large, ou "personnels ${ }_{2}$ ", que nous tiendrons ici pour être l'ensemble regroupant les personnels, les possessifs (personnels) forts mine, yours, etc. et les réfléchis myself, yourself, etc.?

32 La reconnaissance d'une classe plus large de pronoms personnels ${ }_{2}$ est motivée par le fait que les pronoms concernés partagent certaines propriétés formelles et fonctionnelles fondamentales. Morphologiquement, ils portent des marques de personne $(1,2,3)$ qui leur valent leur appellation, mais aussi les mêmes marques de nombre et, à la 3e personne du singulier, de genre (m., f., n.). S'ajoutent des ressemblances formelles évidentes entre ces pronoms (ex. me, my, myself et la forme non standard "meself). En revanche, on peut estimer que seuls les personnels ${ }_{1}$ portent également des marques de cas, bien que les possessifs (y compris les possessifs faibles my, your, etc.) soient souvent présentés comme des pronoms personnels au cas génitif.

D'un point de vue syntaxique, les pronoms personnels, sont des sortes de noms qui ne prennent pas de déterminant et sont, plus généralement, employés seuls, constituant ainsi des GN réduits à leur noyau (cf. \$2.2). D'un point de vue fonctionnel, enfin, ce sont tous des pronoms définis qui s'emploient typiquement de façon déictique (pour ceux de 1e ou $2 \mathrm{e}$ p.) ou anaphorique-cataphorique (pour ceux de $3 \mathrm{e}$ p.). On notera toutefois que ce n'est pas le cas du it dit impersonnel ou explétif (ex. It's raining), sans parler des innombrables expressions plus ou moins figées qui contiennent it (ex. You're going to get it / Congratulations: you've made it!). Ce n'est pas le cas non plus du they dit générique, synonyme de "people " (They say you only fall in love once) ; et l'on peut s'interroger quant au caractère déictique du you dit générique (You never know what tomorrow may bring). Cela vaut aussi pour les possessifs et les réfléchis correspondants (ex. your dans You never know what your day is going to bring). 
Si l'on ne peut ignorer les liens qui unissent les pronoms personnels ${ }_{1}$, possessifs et réfléchis, il convient également de reconnaître aux premiers un statut particulier. Les pronoms personnels sont beaucoup plus fréquents que (tous) les autres pronoms de l'anglais et comptent même parmi les mots les plus fréquents de la langue, alors que les réfléchis sont relativement peu fréquents et que les possessifs forts le sont bien moins encore (contrairement aux possessifs faibles). Les tableaux (1-2) ci-dessous donnent un aperçu des écarts de fréquence relevés dans le British National Corpus (le 15/06/2018) entre les pronoms personnels $s_{1}$ et quelques autres pronoms. Ces écarts sont tous très marqués, certains de façon extrême.

\begin{tabular}{|l|l|}
\hline PERS $_{1}$ & BNC freq. \\
\hline I & 858211 \\
\hline me & 127872 \\
\hline you & 661498 \\
\hline he & 633413 \\
\hline him & 152045 \\
\hline she & 350294 \\
\hline her & 98791 \\
\hline it & 1039368 \\
\hline we & 346949 \\
\hline us & 74234 \\
\hline they & 415668 \\
\hline them & 165536 \\
\hline
\end{tabular}

Tableau (1)

\begin{tabular}{|l|l|}
\hline Form & BNC freq. \\
\hline something & 49652 \\
\hline himself & 28696 \\
\hline someone & 17559 \\
\hline everything & 17554 \\
\hline anyone & 14039 \\
\hline myself & 11874 \\
\hline yourself & 10229 \\
\hline each other & 10202 \\
\hline mine & 6067 \\
\hline everybody & 5825 \\
\hline hisprn & 5263 \\
\hline yours & 4059 \\
\hline one another & 2635 \\
\hline
\end{tabular}

Tableau (2)

Les pronoms personnels semblent également être les plus fréquents dans les langues, de façon générale. Ainsi, note Sasse (1993: 669) : «The occurrence of personal pronouns [i.e. personal ${ }_{1}$ ] is nearly universal. If a language does have a distinct class of pronouns, a set of personal pronouns will be the basic member of this class. »

Toutefois, il faut distinguer ici la 3e personne, ou «non-personne » (Benveniste 1966), des deux autres. Les pronoms de 1e et 2e p. semblent bien être (quasi-)universaux, ce qui se comprend aisément : ils désignent les deux participants ou rôles de l'interlocution que sont le locuteur (1e p.) et l'allocutaire (2e p.) et, comme précédemment noté, tout acte d'énonciation requiert un locuteur et instaure plus ou moins explicitement un allocutaire. ${ }^{8}$ En revanche, de nombreuses langues n'ont pas ou pas vraiment de pronoms personnels de $3 \mathrm{e}$., dont, semble-t-il, l'intégralité des langues indo-européennes à des époques anciennes. Les reconstructions modernes des pronoms personnels du proto-indo-européen et même du proto-germanique ne postulent ainsi que des pronoms de 1e et 2e p. (cf. Bičanová \& Blažek 2014). Pour la 3e p., le grec ancien et le latin classique, par exemple, employaient plutôt des démonstratifs ou d'autres anaphoriques. En anglais, comme dans bien d'autres langues, les pronoms personnels de 3e p. semblent d'ailleurs issus d'éléments démonstratifs ou déictiques, d'où notamment la ressemblance formelle encore observable entre they et this/these, that/those. Incidemment, on notera que les pronoms démonstratifs sont, 
comme les personnels ${ }_{1}$, des pronoms qui sont définis, typiquement anaphoriques ou déictiques, et bien plus fréquents que beaucoup d'autres. Selon les données de Biber et al. (1999, chap. 4), par exemple, seuls certains pronoms personnels ${ }_{1}$ sont plus fréquents que le pronom démonstratif that. La prototypie étant une notion graduée, on peut ainsi estimer que les pronoms démonstratifs sont moins centraux que les personnels ${ }_{1}$ mais plus centraux que bien d'autres, alors que certaines grammaires ne reconnaissent que des déterminants démonstratifs avec des emplois différents, that étant alors un déterminant dans What's that? (cf. \$3). ${ }^{9}$

\subsection{Les pronoms comme sous-classe fermée de noms}

De longue tradition, les pronoms ont été considérés comme formant une partie du discours à part entière, une classe de mots primaire au même titre que les noms ou les verbes. Cette classification demeure très courante, mais elle a pourtant de quoi étonner.

Fonctionnellement, d'une part, les pronoms sont des proformes, mais les autres proformes ne sont pas traitées de la même façon et sont en fait largement ignorées des grammaires traditionnelles. Ainsi, les proformes adverbiales (here, there, now, then, thus, so, likewise, otherwise et wherever, whenever, somewhere, everywhere...), malgré leur nombre, sont généralement considérées comme de simples adverbes. Il ne leur est pas conféré de statut grammatical particulier et elles ne sont même pas regroupées en une sous-classe au sein des adverbes.

39 Grammaticalement, d'autre part, les pronoms sont des sortes de noms. La distribution du nom propre David, par exemple, est similaire à celle du pronom personnel he/him et s'y apparente en fait davantage qu'à celle du nom commun boy. Suivant une analyse commune, les pronoms occupent typiquement la position de noyau du GN, mais, comme les noms propres, sont le plus souvent employés seuls, ne prenant ni déterminant, ni modificateur ou complément, ce qui en fait des équivalents de GN pleins jouant des fonctions dites nominales (sujet, complément d'objet, etc.). ${ }^{10}$ Comme le note également Hudson (2007 : 190), " since noun phrases are defined by their distribution, they must also include phrases headed by pronouns, and so pronouns must also be nouns ». Ce n'est assurément pas là une idée nouvelle : "A pronoun is a Noun » écrivait déjà Butler (1633) à la suite d'autres, tels que La Ramée, qui proposait également cette analyse dans sa Gramere (1562) du français.

Sémantiquement, enfin, on peut considérer que les pronoms sont, comme les noms, des expressions qui dénotent des "choses " (inanimées ou animées, concrètes ou abstraites) au sens que ce terme a dans la grammaire cognitive et qui recouvre toutes sortes de réifications conceptuelles (cf. Langacker 1987, 1990, 1991, 2008). ${ }^{11} \mathrm{La}$ particularité des pronoms par rapport à des noms prototypiques tels que boy et table est ici que ce sont des mots grammaticaux dont le contenu tend à être schématique (abstrait, « léger »), se réduisant plus ou moins à des traits généraux tels que /défini/, / humain/, /inanimé/, /masculin/, /singulier/, etc. De plus, les pronoms forment ensemble une classe fermée, ou un ensemble réduit de sous-classes fermées (personnels ${ }_{1}$, possessifs, réfléchis, etc.), alors que les noms communs et propres forment des classes lexicales ouvertes.

41 Anticipant quelque peu sur la troisième partie de cet article, on notera également ici que les déterminants, qui sont si étroitement liés aux pronoms que certains ne les en 
distinguent pas, peuvent eux aussi êtres considérés comme une classe fermée d'expressions nominales (cf. §3.1).

Pour ces diverses raisons, en place d'un système des parties du discours dans lequel les noms, les pronoms et les déterminants sont trois classes primaires distinctes, on pourrait envisager un système dans lequel une "super-classe » des noms regrouperait ces divers types de mots et serait scindée en deux sous-classes : (i) les noms lexicaux, regroupant les noms propres et les noms communs (classes ouvertes) ; (ii) les noms grammaticaux, regroupant les déterminants et les pronoms (classes fermées), comme le suggère la Fig. 1. D'un point de vue descriptif, cette réorganisation présenterait certains avantages : elle permettrait, par exemple, de rendre compte des nombreux liens qui unissent les déterminants et les pronoms, ce que ne fait aucunement le modèle standard des parties du discours, tout en maintenant une distinction entre ces deux (sous-)classes ; elle permettrait également de rendre compte des propriétés nominales des pronoms et des déterminants tout en les distinguant des noms propres et des noms communs et en maintenant le regroupement traditionnel de ces deux derniers.

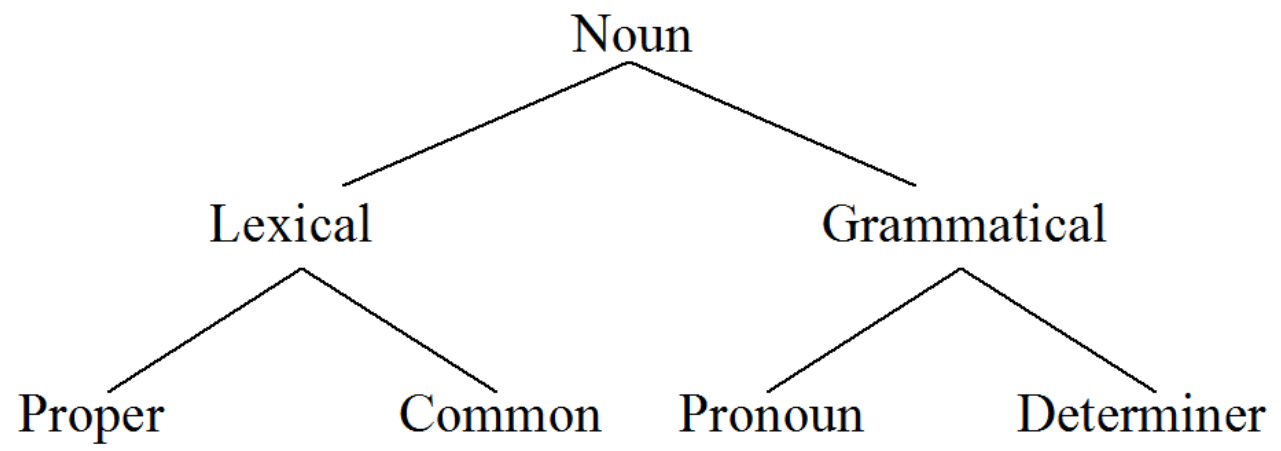

Figure $1:$ La « super-classe » des noms

\subsection{Absence de déterminant}

Les pronoms centraux ne prennent pas de déterminant et la très grande majorité des expressions habituellement classées parmi les pronoms n'en prend pas non plus : ${ }^{*} a$ you, *the himself, *this hers, *that whom, *an everything, etc. ${ }^{12}$ Poser comme principe que les pronoms ne prennent pas de déterminant confère à la classe une plus grande homogénéité grammaticale. C'est là une raison, mais pas la seule, de ne pas inclure parmi les pronoms des expressions telles que (the) same et (my) own, contrairement à une pratique relativement courante, ou encore one(s) et other(s), qui méritent quelques commentaires.

Par one(s) et other(s), nous entendons ici les variantes de ces mots qui ont une forme plurielle en $-s$ et qu'illustrent notamment les exemples suivants :

(17) a. The other room is just like this one.

b. Do you sell any French ones?

c. The tall girl laughed but the other didn't.

c. Like many others, she was shocked and bewildered.

One(s) et other(s) sont des proformes nominales qui s'emploient typiquement de façon anaphorique et parfois déictique, ce qui vaut généralement à ces mots d'être classés parmi les pronoms, voire plus spécifiquement parmi les pronoms indéfinis (ex. Quirk et 
al. 1985, Biber et al. 1999). Grammaticalement, pourtant, one(s) et other(s) diffèrent des (autres) pronoms et sont essentiellement des noms dénombrables. Il est vrai que certaines particularités les distinguent de noms dénombrables prototypiques : par exemple, de nombreuses combinaisons [D + one $(s)]$ sont acceptables uniquement si one(s) est accompagné d'un modificateur et sont autrement préemptées par d'autres formes, ex. a blue one vs *a one (= one); her old one vs *her one (= hers); some French ones vs *some ones (= some); three funny ones vs *three ones (= three). Quant à other $(s)$, il ne peut être prémodifié que par des numéraux cardinaux et quelques adjectifs de quantité et ses emplois sont fortement concurrencés par [other ${ }_{\text {Adj }}(\operatorname{Adj})$ one $\left.(s)\right]$, ex. * the blue other (= the other blue one), *some tall others (= some other tall ones), *an other (= another (one)), ${ }^{*} a$ good other (= another good one). Pour le reste, cependant, one(s) et other(s) varient en nombre, ayant même un pluriel régulier en -s; ils doivent être déterminés lorsqu'ils sont au singulier $\left({ }^{*}\right.$ Take _ blue one ; ${ }^{*}$ Take _ other) ; ils n'acceptent que des déterminants compatibles avec les noms dénombrables (ex. several ones/others vs * much one(s)/ other(s)) ; et ils sont aussi bien compatibles avec des déterminants définis (this big one; the three others) qu'indéfinis (a good one; several others). Cette dernière propriété est attendue si l'on considère que ce sont des noms dénombrables, mais elle ne l'est pas si l'on considère que ce sont des pronoms, puisqu'on considère habituellement que les pronoms sont tous, soit définis, soit indéfinis. La classification de one(s) et other $(s)$ parmi les pronoms, et plus encore parmi les pronoms indéfinis, paraît donc incohérente. Elle illustre le fait, notamment relevé par Haspelmath (1997 : 11), que la catégorie des pronoms indéfinis s'apparente dans de nombreuses traditions grammaticales à une "catégorie poubelle " ("a waste-basket category »), i.e. une classe (comme aussi celle des adverbes) dans laquelle on range des éléments que l'on ne parvient pas à faire entrer dans les autres, ce qui la rend inévitablement très hétérogène.

Pour ces diverses raisons, il semble donc bien justifié d'exclure one(s) et other(s) de la classe des pronoms : fonctionnellement, ce sont des proformes, mais grammaticalement, ce sont des noms dénombrables, malgré certaines particularités.

\section{Les pronoms et les déterminants}

Les pronoms et les déterminants, si l'on s'en tient à des listes relativement traditionnelles de leurs membres, sont deux classes de mots fermées qui se recouvrent très largement.

On peut ainsi reconnaître de nombreux "couples » déterminant-pronom dont les membres sont identiques ou ne diffèrent que légèrement, formellement et/ou sémantiquement :
(18) a. That's your problem, not \{my problem / mine\}.
b. Can I have one of \{those biscuits / those\}?
c. I need \{another pen / another\}.
d. She got four votes and I got \{three votes / three\}.
e. There is \{no difference / none\}.
f. There isn't \{any difference / any\}.
g. Take \{some sugar / some\}.
h. \{Which book / Which\} do you like best?
i. $\{$ Either key / Either\} will do. 
49 s'interroger. Faut-il, par exemple, considérer que my et mine sont deux lexèmes différents - le déterminant (ou adjectif) possessif my et le pronom possessif mine - ou les deux formes d'un seul et même lexème? Si l'on ne reconnaît qu'un seul lexème, faut-il en faire un déterminant, un pronom, ou encore un membre d'une classe hybride de "déterminants-pronoms ", à l'instar des "pronoms-adjectifs» des grammaires anciennes ? ${ }^{13}$ De plus, faut-il traiter tous ces couples de la même façon?

50 Au problème de la catégorie s'ajoute celui de la fonction. Dans l'analyse standard, il y a deux catégories différentes pour deux fonctions différentes. Le pronom est « indépendant »; il joue la fonction de noyau d'un GN et apparaît typiquement seul, ce qui en fait l'équivalent d'un GN plein. Par opposition, le déterminant est un élément dépendant au sein $\mathrm{du} G \mathrm{GN}$; il apparaît devant un nom (et ses éventuels adjectifs prémodificateurs) et est conçu comme une sorte d'adjoint ou modificateur de la tête $\mathrm{N}$ du GN dont il a pour fonction de « déterminer » certaines propriétés référentielles (ex. défini vs indéfini, spécifique vs générique). Cette conception des déterminants explique qu'ils aient longtemps été considérés comme des adjectifs (déterminatifs), quoique les articles aient souvent fait l'objet d'une classification distincte. En anglais, les déterminants se distinguent toutefois d'adjectifs centraux tels que big, old, blue et strange par diverses propriétés, dont le fait qu'ils tendent à être obligatoires, particulièrement devant un $\mathrm{N}$ dénombrable singulier, ex. *She lives in _ (big) house. C'est prioritairement sur cette base que l'on reconnaît communément une classe de déterminants qui inclut notamment les articles, les démonstratifs et possessifs prénominaux, ainsi que certains quantifieurs et mots en wh-, ex. \{a / the / this / our / whose / which / no / either\} (big) house. Le regroupement de ces mots dans une même classe distributionnelle peut être discuté, mais chaque mot ayant dans l'absolu une distribution unique, tout regroupement distributionnel implique un certain degré d'approximation et dépend donc de l'objectif visé (cf. Harris 1963[1951]). ${ }^{14}$

51 Face à l'analyse standard des déterminants et des pronoms existe une multitude d'analyses concurrentes et, pour ainsi dire, toutes les analyses concevables ont été proposées. Selon les ouvrages consultés, on peut ainsi trouver des classifications différentes de tout ou partie des mots communément classés parmi les pronoms ou les déterminants : 


\begin{tabular}{|c|c|c|c|c|c|c|c|c|}
\hline & ART & PERS $_{1}$ & \multicolumn{2}{|c|}{ POSS } & \multicolumn{2}{|c|}{ DEM } & \multicolumn{2}{|c|}{ QUANT } \\
\hline & the book & I love it & my book & it's mine & this book & take this & some tea & have some \\
\hline $\begin{array}{l}\text { Biber et al. } \\
(1999), \\
O E D, \\
L D O C E, \\
C A L D^{15}\end{array}$ & DET & PRN & DET & PRN & DET & PRN & DET & PRN \\
\hline $\begin{array}{l}\text { Bloomfield } \\
\text { (1933) }\end{array}$ & $\mathrm{ADJ}_{\mathrm{det}^{16}}{ }^{16}$ & PRN & \multicolumn{2}{|c|}{$\mathrm{ADJ}_{\mathrm{det}}$} & $\mathrm{ADJ}_{\mathrm{det}}$ & PRN & $\mathrm{ADJ}_{\mathrm{det}}$ & PRN \\
\hline $\begin{array}{l}\text { Quirk et al. } \\
\text { (1985) }\end{array}$ & DET & PRN & \multicolumn{2}{|c|}{ PRN } & DET & PRN & DET & PRN \\
\hline $\begin{array}{l}\text { Payne \& } \\
\text { Huddleston } \\
\text { (2002) }\end{array}$ & DET & PRN & \multicolumn{2}{|c|}{ PRN } & \multicolumn{2}{|c|}{ DET } & \multicolumn{2}{|c|}{ DET } \\
\hline $\begin{array}{l}\text { Jespersen } \\
(1924), \\
\text { Hudson } \\
(1990)\end{array}$ & PRN & PRN & \multicolumn{2}{|c|}{ PRN } & \multicolumn{2}{|c|}{ PRN } & \multicolumn{2}{|c|}{ PRN } \\
\hline $\begin{array}{l}\text { Radford } \\
\text { (2009) }\end{array}$ & $\mathrm{DET} / \mathrm{Q}^{17}$ & DET & \multicolumn{2}{|c|}{ DET } & \multicolumn{2}{|c|}{ DET } & \multicolumn{2}{|c|}{ Q } \\
\hline
\end{tabular}

Tableau (3) - Exemples de classification des articles (ART), pronoms personnels au sens étroit (PERS $)_{1}$, possessifs (POSs), démonstratifs (DEM) et de certains quantifieurs (QUANT)

Nous examinons ci-dessous certains des arguments qui ont été invoqués pour remettre en question la distinction déterminant vs pronom, que ce soit intégralement ou seulement partiellement. Nous arguons ici en faveur du maintien de cette distinction, particulièrement au regard des articles et des pronoms personnels $s_{1}$ tout en reconnaissant qu'elle peut s'avérer parfois délicate, voire incertaine.

\subsection{La distinction catégorielle déterminant vs pronom}

D'un point de vue sémantique, on peut estimer que les mots communément nommés déterminants, à commencer par les articles et les éléments distributionnellement similaires, sont, comme les pronoms, des mots qui dénotent des " choses ", au sens que ce terme a dans le cadre de la grammaire cognitive (cf. n. 11). Autrement dit, on peut estimer que les déterminants sont des expressions nominales et plus précisément des noms que l'on pourrait dire grammaticaux ou fonctionnels par opposition aux noms lexicaux. Que ce soit dans Look at this ou Look at this man, par exemple, this semble « montrer » une chose particulière - un référent spécifique - qui est identifiable dans la situation de référence et proche de l'énonciateur. Dans Look at this, la chose est uniquement montrée et son identification repose sur l'interprétation du seul démonstratif, quoique ce "geste verbal» soit normalement accompagné d'un geste physique de désignation ; dans Look at this man, la chose est à la fois montrée et catégorisée : this désigne la chose et man en spécifie le type - en tant que nom dénombrable singulier, $\operatorname{man}_{\mathrm{sg}}$ désigne plus exactement une instance du type d'entité dénoté par le lexème MAN, et non le type lui-même. ${ }^{15}$ Par suite, la relation entre this et man dans this man, et plus généralement entre $\mathrm{D}$ et $\mathrm{N}$ au sein d'un syntagme du type [D $\mathrm{N}$ ], s'apparente à une forme d'apposition de $\mathrm{N}$ à $\mathrm{D}$, tout en se distinguant à plusieurs égards d'une apposition canonique telle que my friend Peter - D est une catégorie fonctionnelle ; my friend ne détermine pas le nom (propre) Peter ; dans bien des cas, [D $\mathrm{N}$ ] ne peut se réduire, ni à $\mathrm{D}$, ni à $\mathrm{N}$; etc. Cela pourrait en partie expliquer pourquoi il est difficile d'identifier une tête (unique) dans une construction du type [D N], certains considérant que $\mathrm{D}$ dépend de $\mathrm{N}$ - l'analyse GN classique, dans laquelle $\mathrm{D}$ est un adjoint 
de $\mathrm{N}$ - tandis que d'autres maintiennent que c'est au contraire $\mathrm{N}$ qui dépend de $\mathrm{D}$ l'analyse GD, dans laquelle N complémente D (cf. infra). Hudson (2004), qui examine un certain nombre d'arguments pour et contre chacune de ces deux analyses, conclut que $\mathrm{D}$ et $\mathrm{N}$ sont «mutuellement dépendants ", ce qui ne clarifie pas vraiment la situation, mais illustre bien sa complexité.

Etant donné cette analyse de this / this man, on peut se demander s'il est pertinent de distinguer deux this (voire trois, puisque de nombreux ouvrages reconnaissent des adverbes dans It was this/that big) et, de la même façon, deux his, deux some, etc. Il pourrait sembler plus économique, par exemple, de reconnaître un unique pronom this pouvant jouer des fonctions différentes, comme le propose par exemple Jespersen (1924) (cf. infra). De fait, l'analyse pronominale paraitrait appropriée pour this/that big (" as big as this/that »), mais le cas de this/that big man est plus complexe : le déterminant singulier this ${ }_{\mathrm{sg}}$ peut s'employer aussi bien avec un nom humain qu'avec un nom inanimé (I know this man / I know this problem), alors que le pronom this $s_{\text {sg }}$ ne peut normalement pas désigner une personne (??I've talked to this). ${ }^{16}$ Cette différence

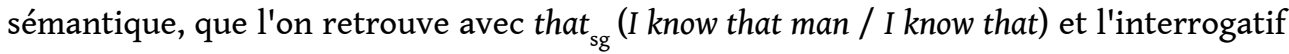
what (What actor do you like best? / What do you like best?), invite plutôt à distinguer deux this qu'à reconnaître deux formes d'un même lexème. Toutefois, d'autres arguments peuvent être avancés et il est difficile de trancher clairement en faveur d'une analyse unitaire ou duelle des démonstratifs. ${ }^{17}$

La distinction catégorielle classique déterminant vs pronom repose en partie sur ces légères différences sémantiques (ex. that boy vs that) ou formelles (ex. my vs mine). Elle repose aussi et surtout sur le postulat d'une différence fonctionnelle (adjoint de $\mathrm{N}$ vs noyau du GN) et sur le fait que certains des mots concernés semblent limités à un seul type d'emploi, à commencer par les représentants les plus fréquents et les plus fondamentaux de chacune des deux classes, i.e. les articles (déterminants prototypiques) et les pronoms personnels ${ }_{1}$ (pronoms prototypiques) :

(19) a. *Read $\{a /$ the $\}$ _..

b. *Read every _- .

c. *the boy [whose _- was lost]

d. *Read [it message]

d'. *[I/you/he director] complained.

e. *Read [myself message].

f. *Read [something/nobody message].

Les articles (19a), mais aussi every (19b) et le relatif whose (19c), sont limités à un emploi prénominal, tandis que les pronoms personnels ${ }_{1}\left(19 \mathrm{~d}-\mathrm{d}^{\prime}\right)$, de même notamment que les réfléchis (19e) et les indéfinis composés something, anybody, everyone, nothing, etc. (19f) semblent limités à un emploi pronominal. Concernant les pronoms personnels, on notera toutefois l'existence de structures du type $\left[w e / u s / y o u_{\mathrm{pl}}+\mathrm{N}_{\mathrm{pl}}\right]$, ex. If only we humans were less ambitious / They used to give us kids chocolates / You girls should know better (BNC). Pour certains, ces structures montreraient que we/us et you $\mathrm{pl}_{\mathrm{pl}}$ ne sont pas des pronoms mais des déterminants (Postal 1966) ou bien qu'il existe des déterminants homonymes des pronoms de 1e et 2e p. du pluriel (Payne \& Huddleston 2002). En anglais standard, les autres pronoms personnels sont exclus de cette construction familière dont l'analyse est discutable. ${ }^{18}$ La prosodie de /'you 'girls/, par exemple, est bien différente de celle d'un GN canonique tel que /the 'girls/, ce qui tend à soutenir l'analyse appositive traditionnelle (cf. Puckica 2013). On peut également penser, de 
façon plus nuancée, que cette construction illustre la possibilité d'un glissement progressif de l'apposition à la détermination.

Les approches unitaires généralisées des " déterminants/pronoms ", i.e. les approches qui réfutent toute distinction catégorielle, présentent toutes certaines difficultés qui invitent à maintenir une distinction entre au moins certains déterminants et certains pronoms, dont les articles et les pronoms personnels ${ }_{1}$. Comme le suggère le tableau $n^{\circ} 3$ supra, lorsqu'une distinction catégorielle est reconnue, elle implique au moins les articles et les pronoms personnels ${ }_{1}$, le statut des autres déterminants/pronoms étant plus variable.

On peut reconnaître deux grands types d'approches unitaires généralisées des déterminants/pronoms, selon qu'elles reconnaissent ou non la distinction fonctionnelle classique entre le déterminant (adjoint du noyau N) et le pronom (noyau du GN). Il y a d'abord celles qui reconnaissent cette distinction fonctionnelle, mais invoquent ce que l'on pourrait nommer le principe de polyfonctionnalité des lexèmes, i.e. le principe suivant lequel les instances d'un lexème peuvent typiquement jouer des fonctions grammaticales différentes (ex. horse dans a horse et $a$ horse dentist). Ainsi, pour Jespersen (1924: 84, 96 sq.), les déterminants (qu'il nomme adjectifs) sont des pronoms et les pronoms peuvent occuper des «rangs fonctionnels » différents : (i) premier rang ou rang primaire (primary rank), i.e. tête d'un GN (ex. Take this / Have some) ; (ii) rang secondaire (secondary rank, adjunct), i.e. adjoint à un mot de rang primaire (this letter / some fun) ; (iii) rang tertiaire (tertiary rank, subjunct), i.e. adjoint à un mot de rang secondaire (ex. It was this/that big). Le principe de polyfonctionnalité des lexèmes n'est pas problématique en soi, mais son application à l'ensemble des déterminants implique notamment que les articles deviennent des pronoms limités à un emploi prénominal, ce qui peut laisser dubitatif. Le postulat de catégories mixtes telles que les "pronomsadjectifs » et autres « déterminants-pronoms » n'est pas plus avantageux : d'un point de vue descriptif, par exemple, faire de the un déterminant-pronom qui n'a jamais d'emploi pronominal ne présente qu'un intérêt assez limité.

D'autres auteurs n'invoquent le principe de polyfonctionnalité que pour seulement certains déterminants ou pronoms. Quirk et al. (1985), par exemple, reconnaissent la distinction déterminant vs pronom, mais considèrent que les possessifs faibles sont les formes déterminatives des pronoms possessifs. Payne \& Huddleston (2002 : 410 sq.) adoptent également cette analyse, mais postulent en outre que certains déterminants et adjectifs, ainsi que certains pronoms, peuvent jouer simultanément deux fonctions au sein d'un GN - ce qu'ils nomment un GN « à tête fusionnée » (fused-head NP) : soit déterminant et noyau (ex. these dans Look at these), soit modificateur et noyau (ex. tallest dans She was the tallest). ${ }^{19}$ Ces propositions tendent inévitablement à compliquer la description des déterminants et des pronoms. Suivant Payne \& Huddleston (2002), par exemple, dans Take $\{$ the / this / my\} pen, the, this et my jouent tous trois la fonction de déterminant du GN objet de take, mais the et this sont des déterminants tandis que my est un pronom. Dans Take \{it / this / mine\}, it et this fonctionnent tous deux comme noyau du GN, mais it est un pronom tandis que this est un déterminant ; quant à mine, c'est un pronom qui joue à la fois la fonction de déterminant et noyau du GN, mais qui est uniquement noyau du GN dans des emplois attributifs tels que Don't touch that: it's mine (id., p. 410).

Aux approches unitaires généralisées des déterminants/pronoms qui reconnaissent la distinction fonctionnelle classique s'en ajoutent d'autres qui la réfutent. Elles sont 
globalement fondées sur l'hypothèse que le noyau d'un syntagme tel que the boy est the et non boy, la différence entre les déterminants et les pronoms étant une affaire de complémentation ou de valence. C'est notamment la position défendue par Hudson (1984, 1990), pour qui les déterminants sont des pronoms qui peuvent ou non être complémentés par des noms communs : certains requièrent un complément (ex. $a$, the), d'autres l'excluent (ex. I, it), d'autres le prennent de façon optionnelle (ex. this, some). Ce type d'analyse est mieux connu sous la forme popularisée par Abney (1987), l'hypothèse ou « analyse GD » (DP anaysis). Suivant cette analyse GD, désormais tenue pour acquise par un grand nombre de grammairiens générativistes, le syntagme the boy est un groupe déterminatif (determiner phrase) dans lequel le déterminant the est le noyau occupant la position $D$ et le nom boy son complément, soit $\left[_{D P}\left[{ }_{D}\right.\right.$ the $]\left[_{N P}\left[\begin{array}{l}\text { boy } \\ \text { d, }\end{array}\right.\right.$ en ignorant ici les diverses "projections fonctionnelles " intermédiaires (AgrP, NumP, $Q P, n P$, etc.) dont le nombre et la nature varient selon les auteurs (cf., entre autres, Bernstein 2001 et 2008, P anagiotidis 2002 et 2005, Giusti 2002, Coene \& D'hulst 2003, Alexiadou et al. 2007, Alexiadou 2014).

61 L'analyse de the boy comme étant un syntagme dans lequel boy complémente the parait à plusieurs égards supérieure à l'analyse classique des GN ; elle se rapproche également davantage de l'analyse de this man proposée plus haut si l'on considère l'apposition comme un type d'adjonction (de $\mathrm{N}$ à $\mathrm{D}$ ). On peut cependant estimer que ces autres approches unitaires des déterminants/pronoms n'offrent pas non plus un traitement tout à fait satisfaisant des articles et des pronoms personnels. Dans l'analyse de Hudson (1984, 1990), les articles $a$ et the deviennent des pronoms qui doivent être obligatoirement complémentés par un nom commun - Hudson (2004) est bien plus réservé puisqu'il argue, comme noté plus haut, que $\mathrm{D}$ et $\mathrm{N}$ sont " mutuellement dépendants ». Dans l'analyse GD, les pronoms personnels deviennent typiquement des déterminants complémentés par des «noms vides » (Panagiotidis 2002), I devenant par exemple $\left[{ }_{D P}\left[{ }_{D} I\right]\left[_{N} \varnothing\right]\right]$ dans une notation à nouveau très simplifiée. ${ }^{20}$ Cela implique une prolifération de noms qui semblent non seulement nuls quant à leur forme, mais également nuls quant à leur contenu - il ne peut être question ici d'ellipse nominale (cf. §3.2). Cette hypothèse est également discutable dans la mesure où les pronoms personnels ${ }_{1}$ peuvent être modifiés par des adjectifs (silly me, lucky you, poor him) ou des propositions relatives, restrictives (à la 3e p. seulement, ex. he who laughs last) ou non restrictives (you, who are never late), comme le sont bien plus souvent les démonstratifs (surtout those, ex. those who complained) et les indéfinis (something new ; all I need). Dans She who hesitates, I'm afraid, just has to drink what she's given (BNC), par exemple, tout laisse à penser que la relative who hesitates modifie she et non pas un hypothétique nom vide qui se trouverait après she, ce qui va plutôt dans le sens de l'analyse classique du pronom comme noyau du GN.

\subsection{L'ellipse nominale}

Il est communément admis que le noyau d'un GN peut, dans certaines conditions, subir une ellipse de discours et cette possibilité d'une ellipse nominale tend à brouiller la distinction déterminant vs pronom. ${ }^{21}$ Dès lors, on peut en effet se demander si certains pronoms ne seraient pas des déterminants au sein de GN elliptiques - des GN dont le noyau pourrait être une position vide anaphoriquement liée à quelque antécédent ou 
une proforme nominale phonétiquement nulle, un « représentant zéro » (Bally 1932) ou une " anaphore zéro » (Bloomfield 1933). ${ }^{22}$

Si l'on considère, par exemple, que les segments soulignés dans (20) ci-dessous sont des cas d'ellipse nominale, alors on peut se demander s'il n'en va pas de même pour (21):

(20) a. Many dolphins were caught but the fastest escaped. $[=$ 'the fastest

dolphin(s)']

b. The first song was bad and the second was worse. [= 'the second song']

c. Give positive advice, not negative. (BNC) ['= negative advice']

$\mathrm{d}$. There are two versions of the anthropic principle, the weak and the strong. (BNC)

[= 'the weak version and the strong version']

(21) a. She got four votes and I got three. [" 'three votes']

b. I needed staples, so I bought some. [= 'some staples']

c. I need sugar but there isn't much/enough left. [= 'much/enough sugar']

d. My car was parked in front of hers. [= 'her car']

e. I don't like her old paintings but I like these. [= 'these paintings']

De façon caractéristique, une structure dite elliptique est une structure qui est incomplète relativement à un standard et dont la partie manquante - il peut y en avoir plus d'une - est sous-entendue et peut être sémantiquement récupérée dans le contexte linguistique ou situationnel. ${ }^{23}$ Idéalement, la partie supposée manquante doit aussi pouvoir être formellement restituée ou rétablie dans la structure en question, bien que cette restitution soit souvent malaisée - elle peut sembler lourde, voire difficilement acceptable, tout comme le remplacement d'un pronom anaphorique par son antécédent (cf. \$1.3). Le principe d'une ellipse sans restitution possible est d'ailleurs communément admis dans le cas des subordonnées comparatives, souvent dites elliptiques alors qu'il s'agit typiquement de propositions sémantiquement incomplètes qui ne peuvent pas être formellement complétées : ex. She's not as strong as she looks (*strong) / He is taller than she is (*tall). De plus, lorsque l'ellipse n'est pas textuelle mais situationnelle, il n'y a pas de propos correspondant à la partie supposée omise et la « restitution » de cette dernière devient nécessairement hasardeuse. L'ellipse est donc une catégorie dont les contours sont relativement flous et l'on peut juger utile d'en adopter une conception souple ou graduée (ex. Quirk et al. 1985 : 883 sq.), i.e. de faire de l'ellipse une catégorie prototypique avec des cas centraux ou « stricts » et d'autres plus marginaux ou «lâches », qui ne présentent pas toutes les caractéristiques du prototype - au risque, toutefois, de rendre les limites de l'ellipse difficilement identifiables.

De prime abord, les segments soulignés dans (21) se prêtent facilement à une analyse elliptique. Dans (21a), par exemple, (i) three se comprend comme signifiant « three votes", (ii) le nom votes, qui peut sembler sous-entendu ou manquant, peut être récupéré dans la première partie de la phrase et (iii) ce nom peut être ajouté ou "restitué » après three, dans la position canonique du noyau d'un GN. Plutôt que de postuler un pronom numéral three homonyme du déterminant ou adjectif three (et de dédoubler similairement tous les autres numéraux cardinaux), il peut sembler plus économique de considérer qu'il s'agit là du déterminant numéral three, mais au sein d'un GN dont le noyau serait une position vide ou une proforme nulle anaphoriquement liée à votes (antécédent) : She got four votes and I got three $\left[\mathrm{C}_{-\mathrm{i}} / \varnothing_{\mathrm{i}}\right]$.

Toutefois, l'« analyse déterminative elliptique » des pronoms de la tradition présente au moins deux limites importantes. Premièrement, elle ne s'applique qu'à certains pronoms : dans You know me, par exemple, rien ne justifie a priori de faire de you et me des déterminants au sein de GN ou GD elliptiques. Aucune expression ou entité n'est ici 
sous-entendue, récupérable ou restituable. Comme noté plus haut, suivant l'analyse GD, you et me seraient ici des déterminants complémentés par des "noms vides ", soit par exemple $\left[{ }_{\mathrm{DP}}\left[{ }_{\mathrm{D}} m e\right]\left[{ }_{\mathrm{N}} \varnothing\right]\right]$ dans une notation simplifiée, mais cet hypothétique nom vide ne peut pas raisonnablement être considéré comme un cas d'ellipse nominale au sens défini plus haut.

Deuxièmement, l'analyse elliptique ne s'applique qu'à certains emplois des pronoms pour lesquels elle a été proposée. Les possessifs forts, par exemple se prêtent à une analyse elliptique dans certains cas (22a-b), mais pas dans d'autres (22c-d) :

(22) a. That's your problem, not mine. [= 'my problem']

b. My car was parked in front of hers. [= 'her car']

c. What is mine is yours.

d. I'd like to know what goes on in that head of his.

Dans (22a), mine se comprend comme signifiant « my problem », ce qui peut en suggérer une analyse elliptique, bien qu'il ne soit pas possible d'ajouter à sa suite le nom problem (*mine problem). Idem dans (22b) avec hers (*car). Parmi les possessifs, seul his, dont les formes prénominale et pronominale sont identiques, permettrait cet ajout (ex. That's your problem, not his (problem)), mais il faut a priori adopter un seul et même traitement pour tous. Pour cette raison, Quirk et al. (1985: 891) rejettent l'analyse elliptique des possessifs forts, qui sont pour eux toujours des pronoms, mais ils conservent néanmoins une position nuancée, notant que la distinction entre ellipse et " substitution » est, dans de tels cas, une " affaire de définition ». Bloomfield (1933: 202 sq.) promeut quant à lui l'analyse elliptique et suggère que mine serait la «forme spéciale » que prend l'«adjectif déterminant» possessif my/mine en cas d'ellipse nominale, allomorphie (de conditionnement assez atypique) qui rendrait compte de l'inacceptabilité de *mine problem (et *hers car, etc.). Dans (22c-d), cependant, rien ne semble justifier l'hypothèse d'une ellipse. Dans ces emplois de type attributif ou appositif (après of), les possessifs forts semblent évoquer tout ce qui est inclus dans la "sphère » du possesseur - potentiellement, tout ce que le possesseur « a ", tout ce qui peut être identifié relativement à lui. Aucune expression ou entité n'est sous-entendue, récupérable ou restituable, y compris avec his: that head of his, par exemple, n'est évidemment pas une forme elliptique de *that head of his head(s). L'analyse pronominale de mine, yours, etc. présente ici l'avantage de pouvoir s'appliquer tout aussi facilement à leurs divers emplois.

De la même façon, les numéraux et autres quantifieurs employés " seuls » se prêtent à une analyse elliptique dans certains cas, mais pas dans d'autres :

(23) a. A: Is there any difference? - B: No, there isn't any.

b. A: Is there any difference? - B: No, there's none.

c. A: Is there any difference? - B: Yes, there is some.

d. Much has been accomplished.

e. Little was left to the imagination.

f. You've done enough.

g. $\{$ Three / Some $\}$ of the dolphins escaped.

h. $\{$ Much / Little $\}$ of the cake was left.

Dans (23a-c), any, none et some peuvent sembler elliptiques, avec toutefois un problème similaire à celui posé par les possessifs forts, puisque *none difference est inadmissible. (Il y a également une différence phonologique entre le some prénominal, qui peut être faible /səm/ ou fort /s $\Lambda \mathrm{m} /$, et le some pronominal, qui est toujours fort.) Dans (23d-f), en revanche, much, little et enough, qui ont par ailleurs eux aussi des emplois simili- 
elliptiques (ex. 21c), désignent simplement une quantité (inanimée) et aucune expression nominale n'est sous-entendue, récupérable ou restituable.

Les structures partitives (23g-h) sont plus problématiques. Suivant l'analyse classique, par exemple, three est un pronom dans three of the dolphins, étant donné qu'il s'agit d'un emploi « indépendant » du numéral (dans lequel il n'est pas « ajouté » à un nom) et que *three dolphins of the dolphins, comme *some dolphins of the dolphins ou *little cake of the cake, est inacceptable. Cependant, certains adjectifs, dont on ne ferait pas des pronoms, admettent des emplois similaires, tels les numéraux ordinaux ou apparentés (the third/ last of the dolphins), les comparatifs définis (the larger of the two) et les superlatifs (the fastest of the three / the most loyal of my friends), emplois qui ne sont d'ailleurs pas limités à des constructions formellement partitives (ex. 20). ${ }^{24}$ L'analyse elliptique ne semble alors pouvoir être maintenue qu'en postulant une "ellipse obligatoire ", sans restitution possible de l'élément supposé omis. Si l'on écarte à la fois l'ellipse et la conversion (homonymie), une troisième option est d'invoquer la polyfonctionnalité et de postuler un fonctionnement particulier du déterminant ou de l'adjectif : par exemple, une promotion de la fonction de déterminant ou de modificateur à celle de noyau du GN (ex. Halliday \& Hasan 1976) ${ }^{25}$, voire, comme le proposent Payne \& Huddleston (2002), un fonctionnement double, à la fois déterminant et noyau ou à la fois modificateur et noyau (cf. \$3.1 et n. 22). Cette troisième option, toutefois, présente elle aussi des difficultés. Dans (21a) She got four votes and I got three, par exemple, on voit mal comment three pourrait signifier " three votes " si three n'est pas lui-même une proforme anaphorique et si le nom votes n'est aucunement représenté dans le GN, fûtce par une position ou proforme vide anaphoriquement liée à votes. De même, dans (20a) Many dolphins were caught but the fastest escaped, où fastest ne peut a priori être qu'un adjectif, on voit mal comment the fastest pourrait signifier « the fastest dolphin(s) » si le nom dolphin(s) n'est aucunement représenté dans le GN, ni d'ailleurs comment un tel groupe pourrait être grammaticalement singulier ou pluriel (The fastest was/were caught). L'hypothèse de l'ellipse d'un nom singulier ou pluriel paraît alors plus avantageuse.

\section{Conclusion}

72 La classe des pronoms, qui est pour ainsi dire aussi ancienne que la tradition grammaticale occidentale, en demeure également une des classes les plus problématiques.

La définition étymologique du pronom comme mot mis "à la place d'un nom » n'est assurément pas satisfaisante : seuls certains pronoms sont ou peuvent être anaphoriques (ou cataphoriques), d'autres étant typiquement déictiques, et d'autres encore ne pouvant être ni l'un, ni l'autre ; l'antécédent d'un pronom n'est pas nécessairement un nom, ni même une expression nominale; et il est trompeur, voire inapproprié, de dire qu'un pronom anaphorique "remplace " son antécédent, particulièrement lorsque le pronom et son antécédent ne sont pas coréférentiels.

On peut redéfinir la classe des pronoms comme une sous-classe fermée de noms qui ne prennent pas de déterminant et dont les membres centraux fonctionnent typiquement de façon anaphorique(-cataphorique) ou déictique. Cette redéfinition, qui tient compte $\mathrm{du}$ caractère prototypique des pronoms personnels et replace les pronoms au sein de la classe plus large des noms, implique également d'exclure de la classe des pronoms des 
mots tels que one(s) et other(s), qui sont grammaticalement des noms dénombrables. Une proforme, même nominale, n'est pas nécessairement un pronom.

Enfin, la question de la délimitation de la catégorie des pronoms pose inévitablement celle des rapports entre les pronoms et les déterminants. L'existence de nombreux " couples" déterminant-pronom, le principe de polyfonctionnalité des lexèmes, la possibilité d'envisager le déterminant comme noyau d'un GD, ou encore la possibilité d'une ellipse nominale sont autant de facteurs qui tendent à obscurcir la distinction déterminant vs pronom. Toutefois, il y a également des raisons de maintenir cette distinction, particulièrement au regard des articles et des pronoms personnels (au sens étroit), qui tendent à être limités à un seul type de fonctionnement. Abandonner toute distinction catégorielle ou fonctionnelle entre a/the et $I /$ you ne semble pas simplifier pas la description grammaticale. En revanche, on peut considérer que les déterminants sont, comme les pronoms, des sortes de noms grammaticaux, distingués des noms lexicaux. Cette approche permet de maintenir la distinction déterminant vs pronom tout en rendant compte des liens étroits et parfois confus entre ces deux catégories.

\section{BIBLIOGRAPHIE}

Aarts, B., Chalker, S. \& Weiner, E. (2014). Oxford Dictionary of English Grammar. 2nd ed. Oxford: Oxford University Press.

Abney, S.P. (1987). The English Noun Phrase in its Sentential Aspects. Unpublished PhD Dissertation. Cambridge, MA: MIT.

Alexiadou, A., Haegeman, L. \& Stavrou, M. (2007). Noun Phrase in the Generative Perspective. Berlin / New York: Mouton de Gruyter.

Alexiadou, A. (2014) Multiple Determiners and the Structure of DPs. Amsterdam / Philadelphia: John Benjamins.

Arnold, D. \& Spencer, A. (2015). A constructional analysis for the skeptical. In Müller, S. (ed.) Proceedings of the 22nd International Conference on Head-Driven Phrase Structure Grammar, 41-60. Stanford, CA: CSLI Publications.

Bally, Ch. (1932). Linguistique générale et linguistique française. Paris: E. Leroux.

Benveniste, E. (1966). Structure des relations de personne dans le verbe. Première publication dans BSL XLIII, fasc. 1, n¹26, 1946. Reproduit dans Problèmes de linguistique générale, vol. 1, 225236. Paris : Gallimard.

Bernstein, J.B. (2001). The DP hypothesis: Identifying clausal properties in the nominal domain. In Baltin, M. \& Collins, C. (eds.) The Handbook of Contemporary Syntactic Theory, 536-561. Oxford: Blackwell.

Bernstein, J.B. (2008). Reformulating the Determiner Phrase analysis. Language and Linguistics Compass, 2(6), 1246-1270.

Bhat, D.N.S. (2004). Pronouns. Oxford: Oxford University Press. 
Biber, D., Johansson, S., Leech, G., Conrad, S. \& E. Finegan. (1999). Longman Grammar of Spoken and Written English. London: Longman.

Bičanová, L. \& Blažek, V. (2014). Indo-European personal pronouns: Limits of their internal reconstruction. Linguistica Brunensia, 62(1), 29-55.

Bloomfield, L. (1933). Language. New York: Henry Holt \& Co.

Börjars, K. \& Burridge, K. (2010). Introducing English Grammar. 2nd ed. London / New York: Routledge.

Butler, Ch. (1633). The English Grammar. Oxford: W. Turner

Coene, M. \& D'hulst, Y. (eds.) (2003). From NP to DP, Vol. 1: The syntax and semantics of noun phrases. Amsterdam / Philadelphia: J. Benjamins.

Corblin, F. (1995). Les formes de reprise dans le discours. Anaphores et chaînes de référence. Rennes: Presses Universitaires de Rennes.

Cowan, R. (2008). The Teacher's Grammar of English. Cambridge: Cambridge University Press.

Craenenbroeck, J. van \& Temmerman, T. (eds.) (2018). The Oxford Handbook of Ellipsis. Oxford: Oxford University Press.

Gardes Tamine, J., Gautier, A., Mercier-Leca, F. Niklas-Salminen, A. \& Verjans, T. (2015). Cours de grammaire française. Paris: A. Colin.

Giusti, G. (2002). The functional structure of noun phrases: A bare phrase structure approach. In Cinque, G. (ed.) Functional Structure in DP and IP, 54-90. Oxford: Oxford University Press.

Greenbaum, S. (1996). The Oxford English Grammar. Oxford: Oxford University Press.

Günther, C. (2013). The Elliptical Noun Phrase in English: Structure and Use. New York \& London: Routledge.

Haegeman, L. \& Guéron, J. (1999). English Grammar: A Generative Perspective. Malden, MA \& Oxford: Carlton / Blackwell.

Halliday, M.A.K. \& Hasan, R. (1976). Cohesion in English. London: Longman.

Harris, Z. (1963[1951]). Structural Linguistics. 6th impression. Formerly entitled Methods in Structural Linguistics. Chicago \& London: University of Chicago Press.

Haspelmath, M. (1997). Indefinite Pronouns. Oxford: Clarendon Press.

Huddleston, R.D. \& Pullum, G.K. (eds.) (2002). The Cambridge Grammar of the English Language. Cambridge: Cambridge University Press.

Hudson, R. (1984). Word Grammar. Oxford / New York: B. Blackwell.

Hudson, R. (1990). English Word Grammar. Oxford / Cambridge, MA: B. Blackwell.

Hudson, R. (2004). Are determiners heads? Functions of Language, 11(1), 7-42.

Hudson, R. (2007). Language Networks: The New Word Grammar. Oxford / New York: Oxford University Press.

Jespersen, O. (1924). The Philosophy of Grammar. London: G. Allen \& Unwin Ltd.

Karttunen, L. (1969). Pronouns and variables. In: Binnick, R.I., Davidson, A., Green, G.M. \& Morgan, J.L. (eds.) Papers from the Fifth Regional Meeting of the Chicago Linguistic Society, 108-116. Chicago: University of Chicago Press. 
La Ramée, P. 1972[1562]. Gramere. Genève : Slatkine Reprints.

Lallot, J. (1997). Apollonius Dyscole : De la construction (syntaxe). Vol. 1 : Introduction, texte et traduction. Histoire des doctrines de l'antiquité classique 19. Librairie philosophique J. Vrin : Paris.

Lallot, J. (1998). La grammaire de Denys le Thrace. Traduite et annotée par J. Lallot. 2e éd. revue et augmentée. Paris : CNRS Éditions.

Langacker, R.W. (1987). Foundations of Cognitive Grammar, vol. I: Theoretical Prerequisites. Stanford, CA: Stanford University Press.

Langacker, R.W. (1990). Concept, Image, and Symbol: The Cognitive Basis of Grammar. Berlin / New York: Mouton de Gruyter.

Langacker, R.W. (1991). Foundations of Cognitive Grammar, vol. II: Descriptive Application. Stanford, CA: Stanford University Press.

Langacker, R.W. (2008). Cognitive Grammar: A Basic Introduction. Oxford: Oxford University Press.

Leech, G. (2006). A Glossary of English Grammar. Edinburgh: Edinburgh University Press.

Lester, M. \& Beason, L. (2013). The McGraw-Hill Handbook of English Grammar and Usage. 2nd ed. New York, Chicago, etc.: McGraw Hill.

Lowth, R. (1763). A Short Introduction to English Grammar. 2nd ed. London: Millar \& Dodsley.

Lyons, J. (1977). Semantics, vol. 2. Cambridge: Cambridge University Press.

Maas, P. (ed.) (1911). Apollonius Dyscolus. De pronominibus. Bonn: A. Marcus \& E. Weber

McCawley, J.D. (1982). Parentheticals and discontinuous constituent structure. Linguistic Inquiry, 13(1), 91-106.

Murray, L. (1826). English Grammar. Philadelphia: Marot \& Walter.

Panagiotidis, P. (2002). Pronouns, Clitics and Empty Nouns: 'Pronominality' and licensing in syntax. Amsterdam / Philadelphia: J. Benjamins.

Panagiotidis, P. (2005). Determiner heads as arguments and the Pronominal Argument (macro)parameter. In Stavrou, M. \& Terzi, A. (eds.) Advances in Greek Generative Syntax. Amsterdam / Philadelphia: J. Benjamins.

Payne, J. \& Huddleston, R. (2002). Nouns and noun phrases. In Huddleston, R. \& Pullum, G. (eds.), chap. 5 .

Postal, P.M. (1966). On so-called pronouns in English. In Dinneen, F.P. (ed.) Report of the Seventeenth Annual Round Table Meeting of Linguistics and Language Studies. Washington, D.C.: Georgetown University Press.

Puckica, J. (2013). The English possessives and the Determiner v. Pronoun distinction. Cercles 29, 67-97.

Quirk, R., Greenbaum, S., Leech, G. \& Svartvik, J. (1985). A Comprehensive Grammar of the English Language. London: Longman.

Radford, A. (2009). Analysing English Sentences: A Minimalist Approach. Cambridge: Cambridge University Press.

Riegel, M., Pellat, J.-C. \& Rioul, R. (2009). Grammaire méthodique du français. 4e éd. Paris: Quadrige / PUF. 
Sasse, H.-J. (1993). Syntactic categories and subcategories. In: Jacobs, J., Stechow, A. von, Sternefeld, W. \& Vennemann, T. (eds.) Syntax: Ein internationales Handbuch zeitgenössischer Forschung / An International Handbook of Contemporary Research, Vol. 1, 646-686. Berlin / New York: Walter de Gruyter.

Schwabe, K. \& Winkler, S. (eds.) (2003). The Interfaces: Deriving and interpreting omitted structures, 126. Amsterdam / Philadelphia: John Benjamins.

Stirling, L. \& Huddleston, R. (2002). Deixis and Anaphora. In Huddleston, R. \& Pullum, G. (eds.), chap. 17.

Sweet, H. (1892). A New English Grammar, Logical and Historical. Part I: Introduction, Phonology, and Accidence. Oxford: Clarendon Press.

Taylor, J.R. (2000). Possessives in English: An Exploration in Cognitive Grammar. Oxford: Oxford University Press.

Teschner, R.V. \& Evans, E.E. (2007). Analyzing the Grammar of English. 3rd ed. Georgetown: Georgetown U.P.

Trask, R.L. (1993). A Dictionary of Grammatical Terms in Linguistics. London / New York: Routledge.

\section{NOTES}

1. Le grec ancien $\dot{\alpha} v \tau \omega v v \mu i ́ \alpha$, litt. " antonyme », ne doit pas être pris au sens moderne (" mot de sens contraire »), qui date de la seconde moitié du 19e s. Le préfixe $\alpha \dot{\alpha} v(\mathrm{l})$ - n'y évoque pas tant l'opposition que l'échange et la réciprocité, comme peut le faire contre en français dans certains emplois (ex. contrepartie ; échanger une chose contre une autre).

2. Paradoxalement, le terme grec ó $v \tau \omega v v \mu i ́ \alpha$ pourrait d'abord avoir été forgé pour rendre compte du fonctionnement des pronoms de 1e et 2e p. (cf. Lallot $1998: 198-199)$. Ainsi, explique Apollonios Dyscole : «Si les pronoms remplacent les noms [...] c'est que, à la première et à la deuxième personnes, il est impossible d'employer les noms » (Synt. II, §40 / Lallot 1997 : 158). Les pronoms suppléent à la « déficience » des noms (Synt. II, \$2 / Lallot 1997 : 148), car un nom est grammaticalement toujours une 3e p. et ne peut donc pas être employé, par exemple, en position de sujet d'un verbe conjugué à la 1e ou 2e p. (comme en français, ex. \{Vous / ${ }^{*}$ Marie\} allez rire). Il faut dans ce cas employer un pronom (de 1e ou $2 \mathrm{e}$ p.) dont on peut alors également dire qu'il remplace le nom d'une des deux " personnes » qui participent à l'échange verbal. On peut voir là toute l'ambiguïté de l'appellation ơ $v \tau \omega v v \mu$ í $\alpha /$ pronom et toute la subtilité d'Apollonios.

3. En français, proforme semble assez peu usité et on trouve souvent pronom(inal) dans un sens élargi : ainsi, en et $y$ sont parfois nommés pronoms adverbiaux ou adverbes pronominaux. Jespersen (1924 : 84) utilise également l'étiquette pronominal adverb pour then, there, when, where, thus, so, etc.

4. Quirk et al. (1985) évoquent systématiquement " the same » et non simplement "same " pour ces exemples. Ce détail mérite d'être noté, car the same n'est pas un mot, mais un groupe de mots, et ne peut donc pas être un pronom, à proprement parler : same peut y être modifié, quoique de façon très limitée, au moyen de very, exact ou d'expressions plus pittoresques comme bloody, freakin', etc., ou encore suivi d'une proposition relative.

5. (11b) peut être jugé (marginalement) acceptable s'il y a deux personnes différentes nommées Jenny (ou dans un cas de dédoublement de la personnalité, etc.), donc dans une autre interprétation que celle marquée les indices identiques ( “ ${ }_{i}$ ). Même alors, toutefois, l'énoncé demeure difficilement compréhensible et très atypique. 
6. « Antecedent: a unit to which a word or phrase refers back grammatically 》 (Aarts et al. 2014, S.v.). « The word the pronoun refers to is called an antecedent. » (Lester \& Beason $2013: 160$ ) ; « in [...] Mark is always talking to himself, the reflexive pronoun refers to the subject, Mark " (Cowan $2008: 288$ ).

7. Dans cet article, sauf indication contraire, les seuls possessifs considérés sont les possessifs personnels, qui n'incluent pas whose. En anglais, la classe des pronoms dits personnels est généralement limitée aux seuls personnels ${ }_{1}$, mais elle inclut parfois également : (i) les possessifs forts (ex. Lowth 1763, Murray 1826) ; (ii) les possessifs forts et faibles (ex. Quirk et al. 1985) ; (iii) les deux séries de possessifs et les réfléchis (ex. Teschner \& Evans 2007) ; (iv) pour Payne \& Huddleston (2002), les possessifs, les réfléchis, mais aussi l'explétif there (Is there a problem?) et le pronom générique one (One never knows), qui n'est pourtant ni défini, ni " personnel », ni anaphorique ou déictique.

8. L'existence de pronoms personnels (y compris de 1e et 2e p.) est controversée dans certaines langues d'Asie du Sud-Est telles que le birman, le japonais et le thaï : pour désigner le locuteur et l'allocutaire, ces langues utilisent des formes que certains considèrent être des noms et d'autres des pronoms (cf. Bhat $2004: 30-31$ ).

9. C'est notamment la position de Payne \& Huddleston (2002), mais c'est également le cas dans le $\mathrm{BNC}$, d'où l'absence des démonstratifs dans notre tableau $\mathrm{n}^{\circ} 2$.

10. L'analyse du pronom comme tête d'un GN devient plus utile lorsqu'il n'apparaît pas seul : ex. [he [who laughs last]], [[lucky] you], [that [which matters most]], [those [with stripes]], [something [strange]].

11. Dans la définition qu'en donne Langacker (2008 : 136), « a thing is characterized schematically as a set of interconnected entities, grouped and reified to form a unitary entity for higher-level cognitive purposes ».

12. Il est entendu que nous ne considérons pas ici les cas de conversion ou apparentés : ex. Is it a $\underline{\text { he }}$ or a she? / The you that you are now is not the you that you used to be / He is a nobody.

13. La reconnaissance d'une catégorie mixte d'adjectifs-pronoms (ou pronoms-adjectifs) est traditionnelle dans les grammaires du grec ancien ou du latin. Elle est également longtemps demeurée courante dans les grammaires de l'anglais (ex. Sweet $1892: 69)$ et Greenbaum (1996 : 163) propose encore une catégorie "pronoun-determiner».

14. Certains auteurs (ex. Abney 1987, Haegeman \& Guéron 1999, Guisti 2002, Alexiadou et al. 2007) réfutent la validité de ce regroupement, un argument récurrent étant la cooccurrence possible de certains des mots concernés (ex. this our land, structure vieillie et qui semble en fait appositive, comme this the land of my birth), voire même la cooccurrence possible des mots nommés articles, possessifs, etc., dans d'autres langues (ex. it. il tuo amico, litt. 'le tien ami' ; questa mia storia, litt. 'cette mienne histoire').

15. Nous réfutons ici l'analyse courante selon laquelle, dans un syntagme du type [D N], le nom $\mathrm{N}$ dénoterait un type d'entité - une " catégorie ", " classe ", " notion ", etc. (voir, entre autres, Abney (1987 : 76-77), Taylor (2000 : 100) et, pour le français, Riegel et al. (2009: 279)). Dans the book, par ex., book est un nom singulier, qui évoque une instance du type dénoté par le lexème nominal воок, et non le type lui-même, d'où le nombre singulier du syntagme alors que the ne donne aucune indication de nombre. Dans un composé comme book club, en revanche, book est ce qu'on pourrait nommer la forme de base du lexème, qui, elle, dénote bien un type (cf. Langacker $1991: 74$ sq.).

16. Il y a un cas particulier, qui est l'emploi de this comme sujet dans des propositions attributives (This is Joe / Who is this?), mais c'est un emploi également admis par that (Who's that?) ainsi que it (Is it Joe?).

17. Les démonstratifs pluriels peuvent, eux, désigner des personnes dans des emplois qui sont traditionnellement considérés comme pronominaux, ex. I'd rather work with my musicians than 


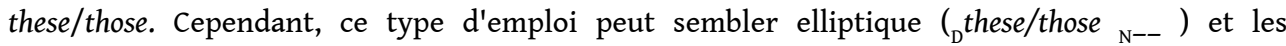
démonstratifs singuliers ne l'admettent normalement pas, y compris avec un antécédent inanimé : dans I'd rather work with my musician/instrument than this/that, le démonstratif seul ne peut pas signifier «this/that musician/instrument» (contrairement à this/that one).

18. On trouve them et même parfois they dans certaines variétés d'anglais, mais cet emploi quasidémonstratif est non standard, ex. "What about them ones? / "Here's how they smokers get their fingers burned (BNC).

19. On peut s'interroger quant à la recevabilité de cette hypothèse de GN à tête fusionnée, qui ne semble avoir rencontré presque aucun écho. Elle contrevient à un principe syntaxique très largement admis, notamment connu sous le nom de single mother condition, suivant lequel un constituant ne peut être immédiatement dominé que par un seul nœud, ce qui revient à dire qu'un mot ne peut pas jouer à la fois deux fonctions différentes dans un énoncé donné (voir cependant McCawley 1982 et Arnold \& Spencer 2015). Par ailleurs, elle ne règle pas certains des problèmes pour lesquels elle est typiquement invoquée par ses promoteurs, à savoir les cas supposés d'ellipse nominale (cf. \$3.2).

20. Dans la thèse de Abney (1987 : 281-4), les pronoms personnels étaient des déterminants sans complément, mais l'idée d'en faire des déterminants complémentés par des noms de forme nulle s'est imposée dans la littérature générativiste au nom d'un principe d'uniformité qui exclut le postulat de têtes fonctionnelles sans complément (cf. Panagiotidis $2002: 13 \mathrm{sq}$.).

21. Par simplicité, nous réduirons ici ellipse nominale à ce sens d'ellipse discursive ou contextuelle du noyau d'un GN. Dans l'analyse GD, l'ellipse du noyau N ou N' d'un GN devient l'ellipse d'un GN enchâssé dans un GD, mais les données du problème considéré ici n'en sont pas fondamentalement modifiées.

22. Nous ne considérerons ici que des cas potentiels d'ellipse textuelle, mais l'ellipse peut également être situationnelle (ex. Don't! / How could you?). Si l'ellipse nominale implique une proforme zéro, cette dernière pourrait donc être anaphorique ou déictique.

23. Voir entre autres Quirk et al. (1985: 883 sq.), Schwabe \& Winkler (2003), Günther (2013) et Craenenbroeck \& Temmerman (2018).

24. Lorsqu'ils sont précédés de the mais ne sont pas suivis d'un substantif, certains de ces adjectifs, dont (the) last, (the) next, (the) former et (the) latter, sont parfois considérés comme des (pro)noms et listés comme tels dans les dictionnaires. On voit pourtant mal pourquoi (the) last ou (the) next, qui indiquent une position unique au sein d'une série, devraient être analysés différemment d'adjectifs numéraux ordinaux tels que (the) third ou (the) twenty-fifth. De même, si (the) former et (the) latter présentent un certain degré de lexicalisation, ils semblent demeurer des adjectifs comparatifs définis. Former, par exemple, ne peut s'employer seul que s'il est accompagné de l'article défini, comme les autres adjectifs comparatifs : ex. She bought the \{former / cheaper\} (of the two) vs * She bought a \{former / cheaper\}.

25. Pour Halliday \& Hasan (1976: 147 sq.), toute « ellipse nominale » est en fait un cas dans lequel un élément qui est normalement subordonné au noyau $\mathrm{N}_{\text {lex }}$ se trouve promu au rang de noyau du GN. 


\section{RÉSUMÉS}

Cet article traite de la catégorie des pronoms, de sa définition et de la distinction entre les pronoms et les déterminants. L'article débute par un examen de la définition traditionnelle et étymologique du pronom comme mot mis "à la place d'un nom ", une définition bien peu adaptée puisque seuls certains pronoms, par exemple, peuvent avoir un fonctionnement anaphorique. Une redéfinition est ensuite proposée, faisant des pronoms une classe fermée de noms grammaticaux qui ne prennent pas de déterminant et dont les membres centraux s'emploient typiquement de façon anaphorique ou déictique. La dernière partie de l'article porte sur la distinction entre les pronoms et les déterminants et certains problèmes qui y sont liés, tels que l'ellipse nominale. Il y est argué que les déterminants sont eux aussi des noms grammaticaux, mais que le maintien d'une distinction déterminant vs pronom est motivé et descriptivement utile au regard d'au moins certains des mots concernés, à commencer par les articles et les pronoms personnels.

This article deals with the pronoun word-class, its definition and the distinction between pronouns and determiners. It first examines the traditional and etymological definition of the pronoun as a word put 'instead of a noun', a definition which is hardly appropriate for various reasons, beginning with the fact that only some pronouns can be used anaphorically. A redefinition of the pronoun class is then proposed as a closed class of grammatical nouns that do not take determiners and whose central members are typically used anaphorically or deictically. The third part of the article focuses on the distinction between pronouns and determiners and related issues such as nominal ellipsis. It is argued that determiners are also grammatical nouns, yet that the distinction between determiners and pronouns is motivated and descriptively useful in at least some cases, most particularly with regard to the articles and the personal pronouns.

\section{INDEX}

Mots-clés : classes de mots, pronom, proforme, déterminant, nom grammatical, ellipse nominale

Keywords : word-classes, pronoun, pro-form, determiner, grammatical noun, nominal ellipsis

\section{AUTEUR \\ JÉRÔME PUCKICA}

Université Grenoble Alpes - LIDILEM 